\title{
Good Practice Guide for calibrating a hydrophone "in situ" with a non-omnidirectional source at $10 \mathrm{kHz}$
}

\author{
Albert Garcia-Benadí ${ }^{1}$, Javier Cadena-Muñoz ${ }^{2}$, Joaquín del Río Fernandez ${ }^{2}$, Xavier Roset Juan ${ }^{2}$, Antoni \\ Manuel-Làzaro ${ }^{2}$ \\ ${ }^{1}$ Laboratori de Metrologia i Calibratge, Centre Tecnològic de Vilanova i la Geltrú, Universitat Politècnica de Catalunya (UPC), Rambla \\ Exposició, 24,08800 Vilanova i la Geltrú, Barcelona, Spain, albert.garcia-benadi@upc.edu \\ ${ }^{2}$ SARTI Research Group. Electronics Dept. Universitat Politècnica de Catalunya (UPC). Rambla Exposició 24, 08800, Vilanova i la Geltrú. \\ Barcelona. Spain.+(34) 938967 200, www.cdsarti.org
}

\section{ABSTRACT}

The aim of this paper is to provide the basis for the calibration of a hydrophone "in situ" with a non-omnidirectional source at $10 \mathrm{kHz}$, thus assigning a value of uncertainty, which may be high, but according to requirements, may be sufficient.

\section{Section: RESEARCH PAPER}

Keywords: hydrophone; uncertainty budget; seawater

Citation: Albert Garcia-Benadí, Javier Cadena-Muñoz, Joaquín del Río Fernandez, Xavier Roset Juan, Antoni Manuel-Làzaro, Good Practice Guide for calibrating a hydrophone "in situ" with a non-omnidirectional source at $10 \mathrm{kHz}$, Acta IMEKO, vol. 4, no. 1, article 6, February 2015, identifier: IMEKO-ACTA-04 (2015)-01-06

Editor: Paolo Carbone, University of Perugia

Received December $10^{\text {th }}, 2013$; In final form December $16^{\text {th }}, 2014$; Published February 2015

Copyright: @ 2014 IMEKO. This is an open-access article distributed under the terms of the Creative Commons Attribution 3.0 License, which permits unrestricted use, distribution, and reproduction in any medium, provided the original author and source are credited

Funding: (none reported)

Corresponding author: Albert Garcia-Benadí, e-mail: albert.garcia-benadi@upc.edu

\section{INTRODUCTION}

Nowadays a multitude of tests in the marine environment are performed, such as the measurement of salinity, acidity or basicity $(\mathrm{pH})$ and carbon dioxide $\left(\mathrm{CO}_{2}\right)$. Some of these tests include the measurement of noise pollution as well as the study of cetaceans in the marine environment. Hydrophones are used to perform such tests. These devices are microphones for the marine environment. The hydrophones can be of different types, but their principal task is the transformation of pressure variation into an electrical variation. Most hydrophones are based on a piezoelectric transducer that generates electricity when exposed to a change in pressure.

The main parameter that relates the electrical and pressure magnitude is sensitivity. The following equation (1) is used to measure sensitivity

$$
S=20 \cdot \log \left(\frac{V / V_{\text {ref }}}{P / P_{\text {ref }}}\right)
$$

The unit of sensitivity is expressed as $\mathrm{dB}$ rel $1 \mathrm{~V} / \mu \mathrm{Pa}$, because its unit is $\mathrm{dB}$ (deciBels) and then the unit details the reference values, in this case $V_{\text {ref }}$ is $1 \mathrm{~V}$ and $P_{\text {ref }} i s \quad 1 \mu \mathrm{Pa}$. The quantity $P_{\text {ref }}$ is $1 \mu \mathrm{Pa}$ because it is the basic pressure in seawater. In air $P_{\text {ref }}$ is $20 \mu \mathrm{Pa}$. In our case the output value is voltage, $\mathrm{V}$, and via the sensitivity the voltage changes to pressure, $P$.

A practical case: the hydrophone has a sensitivity value of $-192 \mathrm{~dB}$ rel $1 \mathrm{~V} / \mu \mathrm{Pa}$, which gives $15 \mathrm{mV}$. The real pressure can be described by the equation (2), and the result is $59 \cdot 106$ $\mu \mathrm{Pa}$. In this example the reference values are not used because the voltage value is calculated in $\mathrm{V}$ and the result obtained is $\mu \mathrm{Pa}$.

$P=\frac{V}{10^{S / 20}}$

In (2) the pressure value is calculated, but the interesting parameter is the Reception Level, RL. Equation (3) shows the relation with the pressure and the result is in $\mathrm{dB}$. 

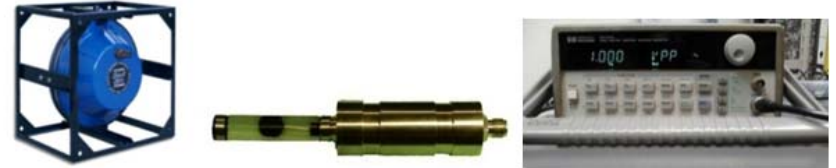

Figure 1. The non-omnidirectional sound pressure generator, Lubell model LL9642T, used as acoustic source (left), the Hydrophone (Bjørge Naxyx Ethernet Hydrophone 02345 used as acoustic receiver (center), and the Signal generator HP33120A (right).

$$
R L=20 \cdot \log \left(\frac{P_{\text {read }}}{P_{\text {reference }}}\right)
$$

The real result for $15 \mathrm{mV}$ is $155.52 \mathrm{~dB}$.

The sensitivity is a function of frequency, thus the aim of the hydrophone calibration is to obtain the sensitivity as a function of frequency.

The calibration of this equipment is detailed in various standards such as [1], where the calibration is done as a function of frequency. The standard calibrations are carried out in laboratories where all parameters are controlled. The calibration method is described in [1]. The standard is divided into 7 topics: Free-field reciprocity, Free-field calibration by comparison, Calibration by hydrostatic excitation, Calibration by piezoelectric compensation, Acoustical coupler reciprocity, Calibration with a pistonphone and Calibration with a vibrating column. The uncertainty value obtained in accredited laboratories is less than $1 \mathrm{~dB}$ rel $1 \mathrm{~V} / \mu \mathrm{Pa}$. Although standard calibrations of hydrophones are essential to maintain the quality of acoustic monitoring, there is also a need for in-situ calibrations. The high cost for marine observatories to retrieve and redeploy the hydrophones, the requirement for continuous operation and the need for more frequent calibrations are the main factors carried out on in-situ calibrations. Therefore, we propose the calibration of the hydrophone in the marine environment at $10 \mathrm{kHz}$ with a non-omnidirectional source. The reason for using a nonomnidirectional source is that it has more problems, and the purpose of the paper is to solve those problems by giving clear guidelines.

This method of calibration involves a considerable increase in the uncertainty because the main parameters are

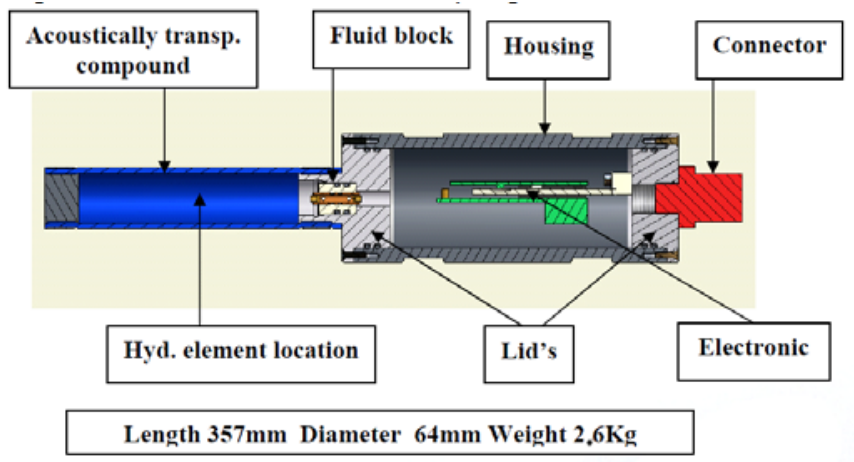

Figure 2. Main components in the hydrophone. not controlled, but measured. Another problem is the low reproducibility of the test since the same conditions over sea surface and underwater will not occur again. However, in many cases this increment of the uncertainty compensates the little investment in performing the calibration. The objective of the calibration is to obtain the sensitivity as a function of frequency.

Performing the calibration in a laboratory is expensive because of the logistic involves three steps. The first is the process which involves ships and divers or ROV (Remote Operated Vehicle) to bring the hydrophone back to the surface. The second step is the processing time for the calibration and the last one is similar to the first step but for the return process to put the equipment back. All these procedures are expensive, and the processing time could be more than 1 month. For these reasons the aim of the paper is to propose an in situ calibration methodology to reduce the calibration time and costs.

In order to achieve this objective it is necessary to evaluate various elements of the environment as well as the spreading factor, the attenuation index and the echo factor.

\section{DEVELOPMENT}

In this section, the method used, the equipment required, the basic equations of the sound level in marine environment, the geometrical approach of the scene, all relevant factors in marine environment sound and their uncertainty calculations will be explained in detail.

\subsection{Equipment}

The equipment that is needed to carry out the calibration consists of a sound source, a hydrophone and a GNSS (Global Navigation Satellite System) receiver.

The properties of the sound source, depicted in Figure 1 (left), have to be known, as well as its calibration uncertainty, the TVR (Transmit Voltage Response) as a function of the SPL (sound pressure level) and the emission frequency.

The hydrophone, depicted in Figure 1 (center), (DUT, Device Under Test) must be characterized with its sensitivity as a function of frequency. In our case, the hydrophone is an integrated device, including the analogue receiver, the digital converters and the Ethernet transmitter. The hydrophone is a Bjorge mark, model Naxys Ethernet 02345. It is composed of an acoustically transparent coverage membrane, where the transducer element is located. Figure 2 shows all enclosed elements.

The signals sent by the hydrophone are received by an internet protocol suite, in this case User Datagram Protocol (UDP) server. The data structure is detailed on the webpage of the manufacturer.

The GNSS receiver has to be able to get the raw data from the satellite and to be compatible with an open source program package such as RTKLIB [2] applications, where the acronym RTK means Real Time Kinetic.

The signal generator used in this calibration procedure is a HP 33120A device. It is depicted in Figure 1 (right) and provides the signal of amplification. It is connected to the 


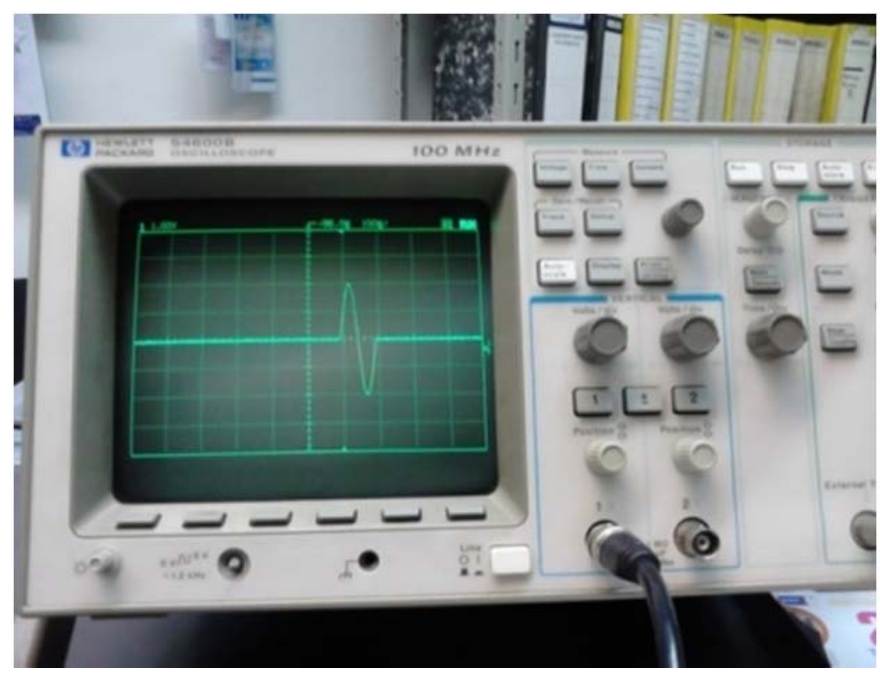

Figure 3. Pulse injected into environment.

amplifier and a sound pressure generator. The signal generated is a pulse with a frequency of $10 \mathrm{kHz}$, which will be generated once per second. Figure 3 shows one of these pulses injected into the environment.

The reason why a pulse is generated every second is to avoid any interferences which may arise in reception. If the signal generated is a continuous pulse, there would be interferences in the reception. These interferences will be a surface seawater and seafloor bounce. The preliminary study of the best conditions is detailed in section 2.5.

The compass is imbedded in a GNSS receiver with Bluetooth connection. The GNSS is a Woxter mark, model BT-TRACER100.

\subsection{Basic equations}

The simplified form of the sound wave propagation in environment equation (4), links three acoustic parameters.

$R L=S L-T L$

The source sound level, SL, is the acoustic spectral density produced by an acoustic source recalculated to a distance of 1 meter. The receipt level, RL, is the acoustic signal obtained with the hydrophone. Transmission loss, TL, is a decrease in the sound level radiated by a target over a distance.

The RL value is obtained with a sensor, and the value SL is obtained at 1 meter with another calibrated hydrophone. The measurements are performed with another hydrophone, in this case the B\&K 8103. The calibration is performed at 1 meter deep so as to avoid the initial perturbations of the transient signal from the generator. The output of all generators is detailed at 1 meter. In this case, the source is non-omnidirectional and for this reason it is very important to know the direction of emission. This factor is studied in sections 2.4 and 5.1.

The last contribution is the transmission loss, which is composed of other factors, as described in equation (5).

$T L=C \cdot \log (r \cdot 1000)+\alpha \cdot r \mp R_{\text {ecbo }}$

where $C$ is the spreading factor, is the attenuation index [3], $r$ is the distance between source and receiver in

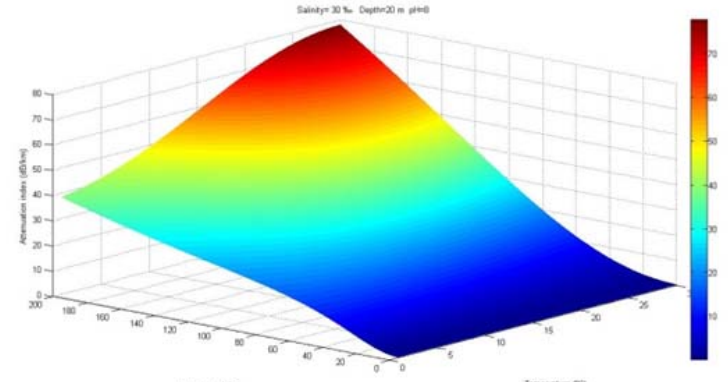

Figure 4. The attenuation index as a function of temperature and frequency. The Salinity, $\mathrm{pH}$ and depth are $30 \%, 8$ and $20 \mathrm{~m}$, respectively.

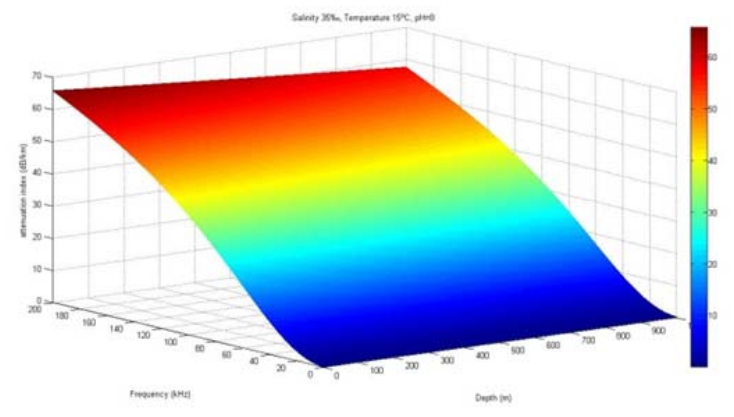

Figure 5. The attenuation index as a function of depth and frequency. The Salinity, $\mathrm{pH}$ and temperature are $30 \%, 8$ and $15{ }^{\circ} \mathrm{C}$, respectively.

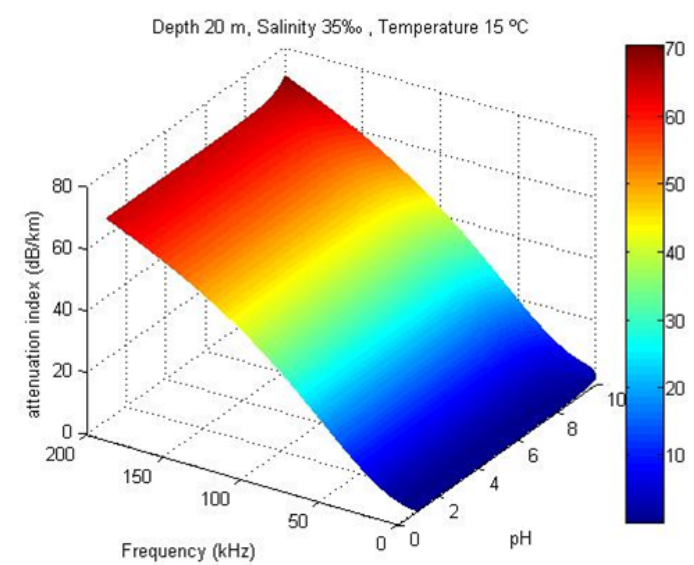

Figure 6. The attenuation index versus $\mathrm{pH}$ and frequency. The Salinity, depth and temperature are $35 \%, 20 \mathrm{~m}$ and $15{ }^{\circ} \mathrm{C}$ respectively.

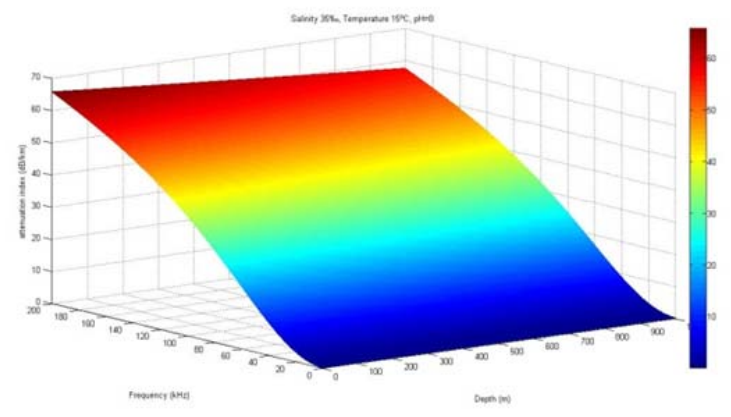

Figure 7. The attenuation index versus salinity and frequency. The depth, $\mathrm{pH}$ and temperature are $20 \mathrm{~m}, 8$ and $15^{\circ} \mathrm{C}$ respectively. 


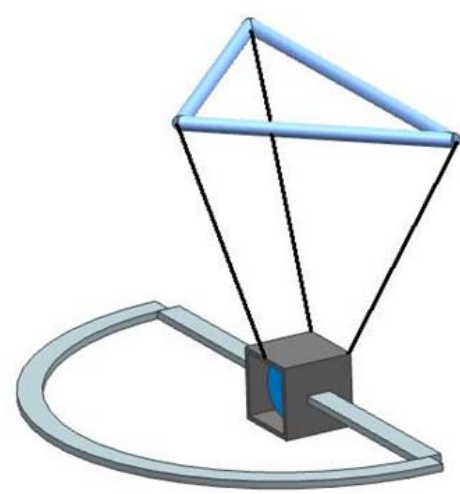

Figure 8. Join scheme of generator, PVC triangle and semicircle.

kilometers, and $R_{\text {echo }}$ is the echo contribution of the seawater surface and the seabed. The bounce in the water surface implies a phase change. However, there is no phase change in the seafloor. As the distance of the first bounce on the water surface is greater than the rebound on the seabed, we consider only one of the two contributions.

Another representation of (4) with the simple variables is (6), where $V$ is the voltage received by the hydrophone, $P_{s}$ is the pressure generated and $P_{0}$ is the basic pressure in the seawater $(1 \mu \mathrm{Pa})$.

$S=20 \cdot \log (V)-20 \cdot \log \left(P_{S} / P_{0}\right)+C \cdot \log (1000 \cdot r)+\alpha \cdot r+R_{\text {echo }}$

The sensitivity, $S$, is ready to be calculated in (3). The terms in (6) can be divided into different fields (7), (8) and (9) from (4), the first one being a function of the receiver, the second a function of the source and the last a function of the environment.

Conceptually the sensitivity is the electrical energy generated minus the pressure energy received, and this pressure energy received is the same as that generated minus the energy absorbed or lost in the environment.

$$
\begin{aligned}
& R L=20 \cdot \log (V)-S \\
& S L=20 \cdot \log \left(P_{S} / P_{0}\right) \\
& T L=C \cdot \log (1000 \cdot r)+\alpha \cdot r+R_{\text {echo }}
\end{aligned}
$$

\subsection{Transmission Loss}

The transmission loss depends on other quantities, as can be seen in equation (5). These factors are detailed below.

The spreading factor is a function of the geometry and relief of the seabed. In an ideal case, the parameter $C$ has two possible values. The $C$ value is 20 for the spherical propagation, and the $C$ value is 10 for the cylindrical propagation. However, in reality the $C$ value is between 10 and 20. This parameter is very difficult to calculate, being the major source of error and uncertainty.

The attenuation index $\alpha$ is a function of other basic parameters, such as the temperature, the salinity, the $\mathrm{pH}$, the depth difference between emission and reception and the emission frequency. The behavior of increasing alpha is due to the increase of the frequency, but the other parameters are also important. The attenuation index $\alpha$

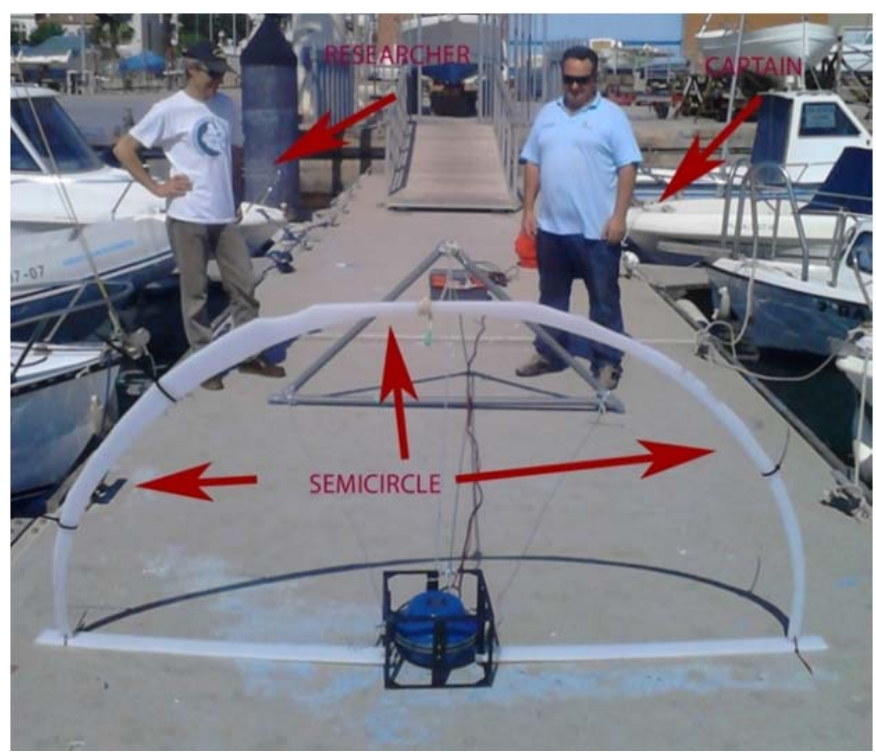

Figure 9. Source generator and nylon semicircle assembly.

depends solely on environmental conditions, and indicates the attenuation value of any waveform that passes through the environment. The alpha value depends on the frequency of this wave, and increases with the frequency of the impulse waveform. For this reason, in the sea you can hear the low frequencies at greater distances than in the air.

Alfa can be modeled as a function of the frequency, $\mathrm{pH}$, depth and temperature by equation (10).

$\alpha=\left(\frac{A_{1} \cdot P_{1} \cdot f_{1}}{f^{2}+f_{1}{ }^{2}}+\frac{A_{2} \cdot P_{2} \cdot f_{2}}{f^{2}+f_{2}{ }^{2}}+A_{3} \cdot P_{3}\right) \cdot f^{2}$

The parameters of equation (10) can be obtained from (11).

$$
\begin{gathered}
A_{1}=\frac{8,86}{c} \cdot 10^{(0,78 \cdot p H-5)} \\
A_{2}=21,44 \cdot \frac{S a}{c} \cdot(1+0,025 \cdot T) \\
A_{3}=4,937 \cdot 10^{-4}-2,59 \cdot 10^{-5} \cdot T+9,11 \cdot 10^{-7} \cdot T^{2}-1,5 \cdot 10^{-8} \cdot T^{3} \\
P_{1}=1 \\
P_{2}=1-1,37 \cdot 10^{-4} \cdot D+6,2 \cdot 10^{-9} \cdot D^{2} \\
P_{3}=1-3,83 \cdot 10^{-5} \cdot D+4,9 \cdot 10^{-10} \cdot D^{2} \\
f_{1}=2,8 \cdot \sqrt{\frac{S a}{35}} \cdot 10^{\left(4-\frac{1245}{T+273}\right)} \\
f_{2}=\frac{8,17 \cdot 10^{\left(8-\frac{1990}{T+273}\right)}}{1+0,0018 \cdot(S a-35)} \\
c=1412+3,21 \cdot T+1,19 \cdot S a+0,0167 \cdot D
\end{gathered}
$$

The variation of $\alpha$ versus frequency and temperature is depicted in Figure 4. The variation of $\alpha$ versus frequency and depth is illustrated in Figure 5. The variation of versus frequency and $\mathrm{pH}$ is shown in Figure 5 and the variation of $\alpha$ versus frequency and salinity is depicted in Figure 7. However, the gradients of salinity and $\mathrm{pH}$ are null, because the study is in offshore. Moreover, because the generator is 


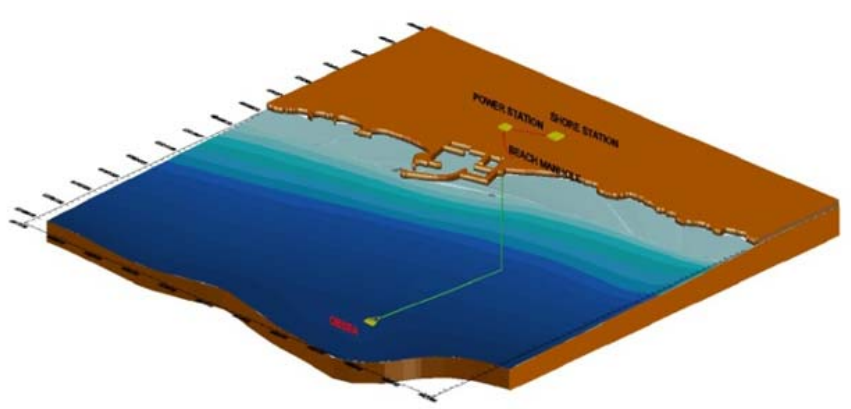

Figure 10. Bathymetry of the Vilanova coast.

located $2 \mathrm{~m}$ under the sea surface, the gradient of temperature is also nil although, the temperature gradient in the first meter under the surface is very high because of the solar radiation.

The last factor is the echo contribution. This factor is very complex to evaluate, thus if in the geometrical scene the velocity of acquisition and the time between two successive sound pulses are well planned, this factor is not nil, but is detectable graphically. For these reasons it only works with the main signal. These factors are described in the section 2.5.

\subsection{Non-omnidirectional source}

The aim of this article is to propose a good practice to evaluate the non-omnidirectional sound marine source, in this case at $10 \mathrm{kHz}$. The first step is to characterize the sound generator as a function of angle at 1 meter of distance. The emitted sound is measured using a calibrated hydrophone, in this case a Brüel \&Kjaer $(\mathrm{B} \& \mathrm{~K})$, model 8103 , located over a semi sphere with a $1 \mathrm{~m}$ radius. Figure 8 shows the semi-circle establishment in the source to obtain the directionality generation of the source.

Given that the source is directional, its directivity is determined by the compass located in the PVC triangle on the water surface.

Initially the mission of the PVC triangle is to prevent the rotation of the generator that is sunk in water, and to determine the direction of emission. Another advantage of the PVC triangle is that it can be used to orient the generator. The generator has a cubic shaped metal skeleton. In the top rear vertices of the cube, the vertices of the PVC triangle are joined, Figure 8.

The B\&K hydrophone is located over the nylon semicircle.

\subsection{Initial Geometrical Study}

The sound velocity in seawater is a function of the salinity, the temperature and the depth, but in this case the tests are made offshore and around $2 \mathrm{~km}$ from DUT position. In these conditions the gradients of temperature, salinity and depth are estimated as being nil because when the temperature and salinity changes, the sound speed changes too. Another important parameter is the seabed relief. In our case the bathymetry illustrated in Figure 10 is known.

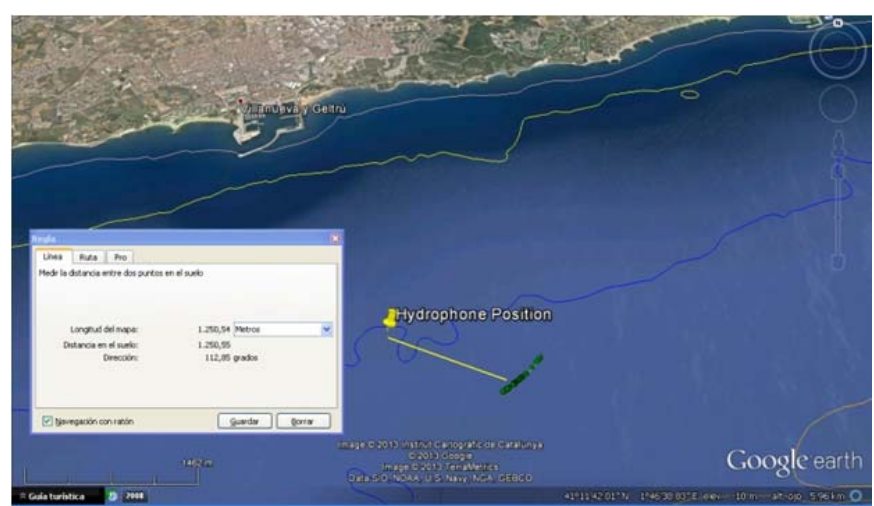

Figure 11. Distance between emission and reception in a real case.

This approach is correct for calculating the distance because the main signal follows a different path than the first echo. Figure 12 shows a schematic with the main parameters.

Equations (12) and (13) are based on the distance, do, which is the minimum distance between the sound generator and the hydrophone, and the elapse time, $t$, between the main wave and its first echo.

$$
\begin{aligned}
& d_{0}=\frac{1}{2 \cdot \sin \theta} \cdot\left[\frac{\left(c \cdot \sin \theta \cdot \Delta t-h_{r}\right)^{2}-3 \cdot h_{r}^{2}}{c \cdot \Delta t \cdot \sin \theta-b_{r}}\right] \\
& \Delta t=\frac{-1}{c \cdot \sin \theta} \cdot\left[\left(b r-d_{0} \cdot \sin \theta\right) \pm \sqrt{d_{0}^{2} \cdot \sin ^{2} \theta-b_{r}^{2}}\right]
\end{aligned}
$$

where:

$b_{r}=$ the distance between the hydrophone and the seafloor

$d_{0}=$ distance between the sound generator and the hydrophone on a plane orthogonal to the surface and including the emitter and the receiver

$c=$ the sound velocity in the water (about $1500 \mathrm{~m} / \mathrm{s}$ )

$\theta=$ angle between the reflected wave and the surface plane in a plane orthogonal to the surface and including the emitter and the receiver

$b_{e}=$ the distance between the generator and the sea surface.

The $b_{e}$ and $b_{r}$ are measured with tape measure. The $b_{r}$ was measured by the diver in the maintenance tasks, and $b_{e}$ was manually measured when the test was done. The $b_{e}$ does not appear because the reflections over the surface are not evaluated since the distance is bigger than $2 \mathrm{~m}$. For this reason, the minimum distance will be $b_{r}$ which is 1 meter.

If we fix the time interval to $1 \mathrm{~s}$, the minimum distance between generator and DUT must be $750 \mathrm{~m}$.

Figure 11 shows an example of the real location used for this test.

\section{PROCEDURE}

The calibration process starts with the generation of the sound pulses through a sound generator, and the reception signal by the hydrophone. The main parameters are the generator position, the environmental conditions of the water, the signal amplitude and its frequency.

The equipment used and the work method applied are detailed in this section. 


\subsection{Readings}

There are two points for collecting the data. The RL is the DUT. The collected data in the ship are: the orientation, measured with a compass situated over the PVC triangle and the GPS satellite signal with the raw signal. The SL is measured with a B\&K hydrophone located over the nylon semicircle. The temperature, the salinity and the $\mathrm{pH}$ are collected through the OBSEA observatory [4] located where the DUT is also positioned.

The readings stored aboard ship are:

- The signal at two frequencies L1 (1575.42 MHz) and L2 $(1227.60 \mathrm{MHz})$ of the reception signal of the GNSS receiver.

- Orientation of the source through a compass located at the main vertices of the triangle.

- Emission depth, $h_{\text {e }}$

The hydrophone collects the following readings:

- Voltage receipt, which is sent to the internet using the protocol UDP (User Datagram Protocol).

- Environmental conditions such as temperature, salinity and $\mathrm{pH}$ are measured in OBSEA.

Other required readings for the post-process are the Institut Català de Cartografia (ICC) caster file that will be included in the free software Real Time Kinetic (RTK). The motivation to use this system is to carry out a differential correction of the position without the need for our own terrestrial base. For this reason, we use the services provided by different entities such as ICC and IGS among others. This allows us to make a high quality positioning but without the financial investment.

\section{UNCERTAINTY CALCULATION}

The uncertainty study starts with (6), where the propagation law is applied by the guide of uncertainty (GUM) [5]. Using the nomenclature detailed in (7), (8) and (9), equation (14) is obtained:

$$
\begin{gathered}
u^{2}(S)=\left(\frac{\partial S}{\partial R L}\right)^{2} \cdot u^{2}(R L)+\left(\frac{\partial S}{\partial S L}\right)^{2} \cdot u^{2}(S L)+\left(\frac{\partial S}{\partial T L}\right)^{2} \cdot u^{2}(T L)= \\
u^{2}(R L)+u^{2}(S L)+u^{2}(T L)
\end{gathered}
$$

In the next subsections the receiver, the generator and the environment uncertainty are reviewed. The terms of all factors included in (14) are not correlated, because the correlation between the distance and the generator (SL), does not exist, since the generation is done irrespective of the distance. The environment is relevant, in fact it is characterized by three parts, the attenuation index

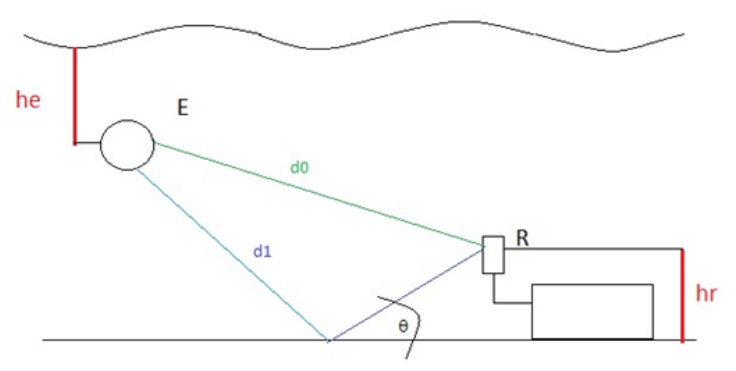

Figure 12 . Schematic test with the main parameters. (independent of distance), the spreading factor (element that depends on the morphology and the depth where the test is performed) and the echo contribution (not considered, since we are at a distance greater than the minimum required).

\subsection{Uncertainty of the receiver}

The hydrophone has been considered as a black box where only output values (counts) are known, because we do not analyze the transducer neither the analog to digital conversion. The elimination of the offset of the signal has been done by the difference between maximum and minimum. Therefore the possible variation of the offset can be eliminated. Thus, the uncertainty of the receiver is a function of the uncertainty in the voltage measurement. The error propagation is shown in (15)

$u^{2}(R L)=\left(\frac{\partial R L}{\partial V}\right)^{2} \cdot u^{2}(V)=\left(\frac{20}{V \cdot \ln (10)}\right)^{2} \cdot \frac{V_{\min }{ }^{2}}{3}$

where the voltage uncertainty has a rectangular probability distribution with a width of the resolution in voltage.

The hydrophone sends the values in counts. The voltage is calculated through the conversion factor of the DAQ and the gain value. In this case, the hydrophone has a converter of 16 bits. As the full scale of the equipment is $5 \mathrm{~V}$, we obtain that the conversion factor between counts and $V$ is $2.5 \mathrm{~V} / 2^{15}$ counts. The value $V_{\min }$ is the minimum voltage that the hydrophone gives and corresponds to the value of 1 count. The value of the squared uncertainty in voltage is $V_{\min } / \sqrt{6}$ corresponding to a rectangular probability distribution with a width equal to the minimum value assigned.

\subsection{Uncertainty of the generator}

The uncertainty of the generator is obtained through its calibration certificate. The error propagation is shown in (16)

$$
u^{2}(S L)=\left(\frac{\partial S L}{\partial P_{S}}\right)^{2} \cdot u^{2}\left(P_{S}\right)=\left(\frac{20}{\ln (10) \cdot P_{S} / P_{0}}\right)^{2} \cdot u^{2}\left(P_{S}\right)
$$

where $u\left(P_{S}\right)$ is obtained in the calibration certificate of the generator as follows (17).

$u^{2}\left(P_{S}\right)=10^{-S_{B K} / 20} \cdot u^{2}\left(V_{B K}\right)+\left(-\frac{V_{B K} \cdot \ln (10)}{20 \cdot 10^{S_{B K} / 20}}\right)^{2} \cdot\left(\frac{U_{G}}{k_{G}}\right)^{2}$

The values of $U_{G}$ and $k_{G}$ are obtained from the calibration certificate of the generator. These parameters are calculated by the accredited laboratory following the standard [1] and the parameter $S_{B K}$ and $V_{B K}$ are the sensitivity and the voltage of the $\mathrm{B} \& \mathrm{~K}$ hydrophones, respectively. This term is included because the output signal is measured with the $\mathrm{B} \& \mathrm{~K}$ hydrophone.

\subsection{Uncertainty of the environment}

The uncertainty of the environment is a function of many parameters and it is the largest contributor to the uncertainty budget. The error propagation is described by equation (18):

$$
u^{2}(T L)=\left(\frac{\partial T L}{\partial \alpha}\right)^{2} \cdot u^{2}(\alpha)+\left(\frac{\partial T L}{\partial r}\right)^{2} \cdot u^{2}(r)+\left(\frac{\partial T L}{\partial C}\right)^{2} \cdot u^{2}(C)
$$




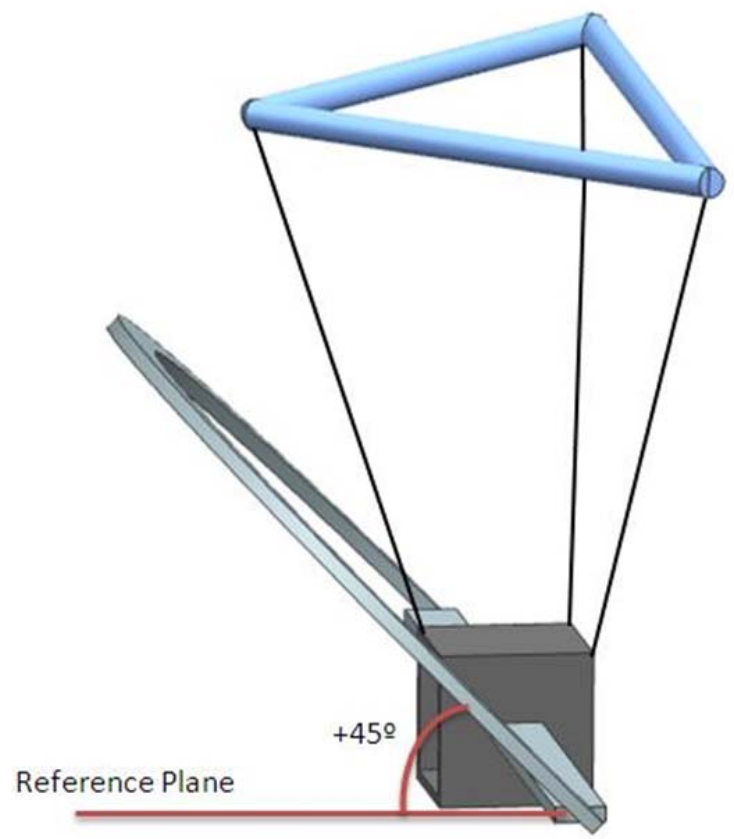

Figure 13. Scheme of the reference plane.

In (18) the uncertainty of $\alpha$, the uncertainty of the distance and the uncertainty of spreading appears. The attenuation index, $\alpha$, is a function of many parameters explained in [3]. Equation (10) shows the dependence of $\alpha$ with other factors.

The values of parameters of equation (10) are detailed in (11), where $c$ is the sound speed in seawater, $S_{a}$ is the salinity in $\%, \mathrm{pH}$ is the measure of the acidity or basicity, $T$ is the temperature in degrees Celsius, and $D$ is the depth in meters. The uncertainty is a function of the typical uncertainty in temperature, salinity depth, and $\mathrm{pH}$. Every term is evaluated through the evaluation of its resolution, its variation and its expanded uncertainty of the calibration certificate with its coverage factor. Equation (19) shows the typical uncertainty, with a fictitious parameter $W$. The parameter $W$ will be finally replaced by the uncertainty of temperature, salinity, $\mathrm{pH}$ and depth.

$u(W)=\sqrt{\left(\frac{W_{\text {resolution }}}{\sqrt{12}}\right)^{2}+\left(\frac{W_{\text {variation }}}{\sqrt{3}}\right)^{2}+\left(\frac{W_{\text {calibratio }}}{k_{\text {calibratio }}}\right)^{2}}$

The spreading uncertainty is calculated using a previous study [6]. This factor is a function of the seabed geometry, the localization and the depth of the generator point, and independent of the other parameters. This factor is a function of the relief of the seabed and the type of generator used.

In order to obtain the uncertainty of the distance, the rectangular probability distribution with a $1 \mathrm{~m}$ width is used. The reception quality factors of the GPS data in raw, after the data integration from the caster, are analyzed offline. We could affirm that the width of the rectangular probability distribution is less $1 \mathrm{~m}$. But this fact is too optimistic; therefore 1 meter is chosen, in this case. It is thus an overestimation.

Some values calculated are detailed in section 5.3.

\section{RESULTS}

\subsection{Direction of emission}

The direction of emission is calculated through the nylon semicircle and the B\&K hydrophone (model 8103); the values obtained are shown in Table 1.

Figure 13 shows the composition graphically.

The heights between the emitter and the receiver remain constant, since neither the emitter nor the receiver changes its height regarding the maximum emission angle and the angle between the DUT position and the PVC triangle indicates, with the compass measure, the correction value to apply.

\subsection{Evaluation of position and distance}

The use of the RTKLib for position correction allows us to know the correct position with an accuracy of, less than $0.5 \mathrm{~m}$. Without this correction the position has an accuracy of around $20 \mathrm{~m}$. Table 1 and Table 2 shows an example of obtained data without correction, and Table 3 shows an example with the corrected data.

Once the emission point is located, then it is necessary to calculate the distance between the generator and the receiver. The distance is calculated with the Vicenty [7] conversion.

Table 4 shows the distance corresponding to the values shown in Table 1.

The uncertainty of distance is very difficult to obtain because the uncertainty for data of GNSS receivers is an independent study, which is being evaluated by many people. In this case, the quality factor included inside the RTKLib has been analyzed and the position has been evaluated with a pair of GNSS.

Once the quality factor of the correction data is evaluated and increased with the contribution of Vicenty conversion, the value of the distance uncertainty is $0.60 \mathrm{~m}$. This value is obtained for a rectangular distribution probability with a width of $1 \mathrm{~m}$.

\subsection{Attenuation index and its uncertainty}

The environment absorption index is calculated with a high-accuracy recorder for conductivity, temperature and pressure (model SBE 16plus V2), henceforth CTD. The parameters are Temperature of 24.04 degrees Celsius, salinity of $38.04 \%$ and $\mathrm{pH}$ of 8.3 . The CTD is located in the OBSEA.

In Table 5, some of the values obtained by the CTD during the tests are shown.

The variations during the test are negligible because of the short interval of the test time of approximately 15 minutes. The attenuation index is $0.85 \mathrm{~dB} / \mathrm{km}$. The effective time has been 15 minutes as the test lasted one hour or more, but not all the positions obtained by the GNSS receiver were reliable. For a position to be reliable it has to be validated with the data obtained by the caster. This does not always happen because satellites change position and sometimes suitable signals or numbers are not available. If the series of data takes a long time to collect, ambient variables in the water would have to be taken into 

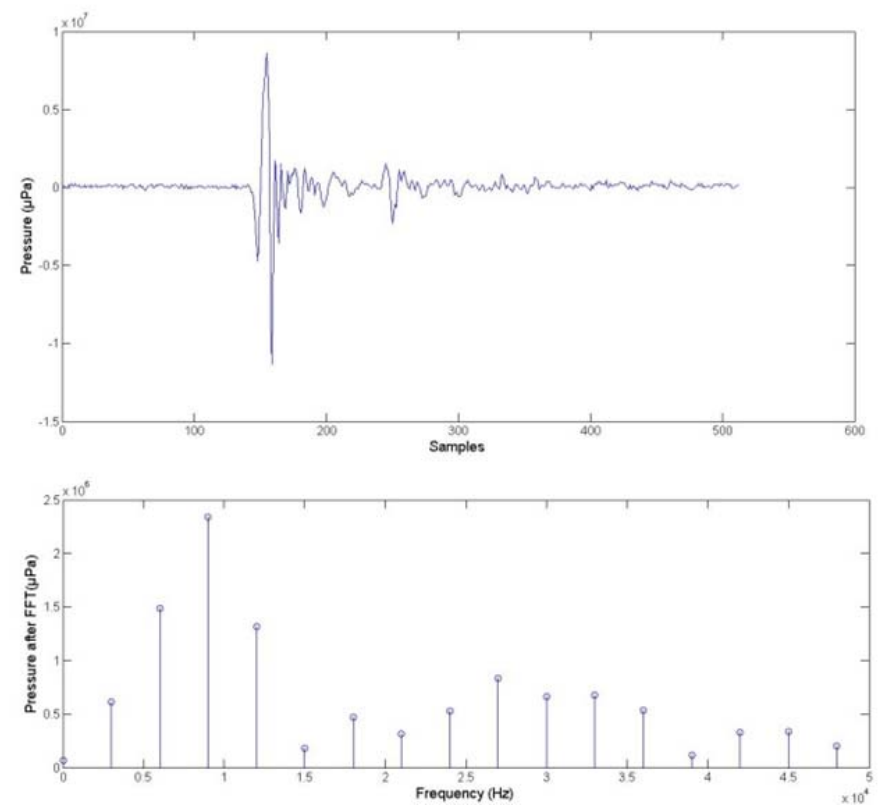

Figure 14. Temporal frame and frequency sub-frame for the acceptation case.

consideration and so there would be a margin of uncertainty at each point. However, in our case, reliable data is taken every 15 minutes, so we can state that the ambient variables such as temperature, $\mathrm{pH}$ and salinity remain constant

The uncertainty value for this case is $0.99 \mathrm{~dB} / \mathrm{km}$. This value is really high in comparison to the absorption index, but it is normal due to uncontrolled parameters.

\subsection{Spreading value}

The spreading value is calculated in another study [6], where the sensitivity uncertainty is known. In this case the value is $16.04 \pm 0.67$. It is important to note that the spreading factor does not have units.

The initial spreading value expected was around 10, because of the shallow depth, about 20 meters. Therefore, a cylindrical propagation is expected. But the change is
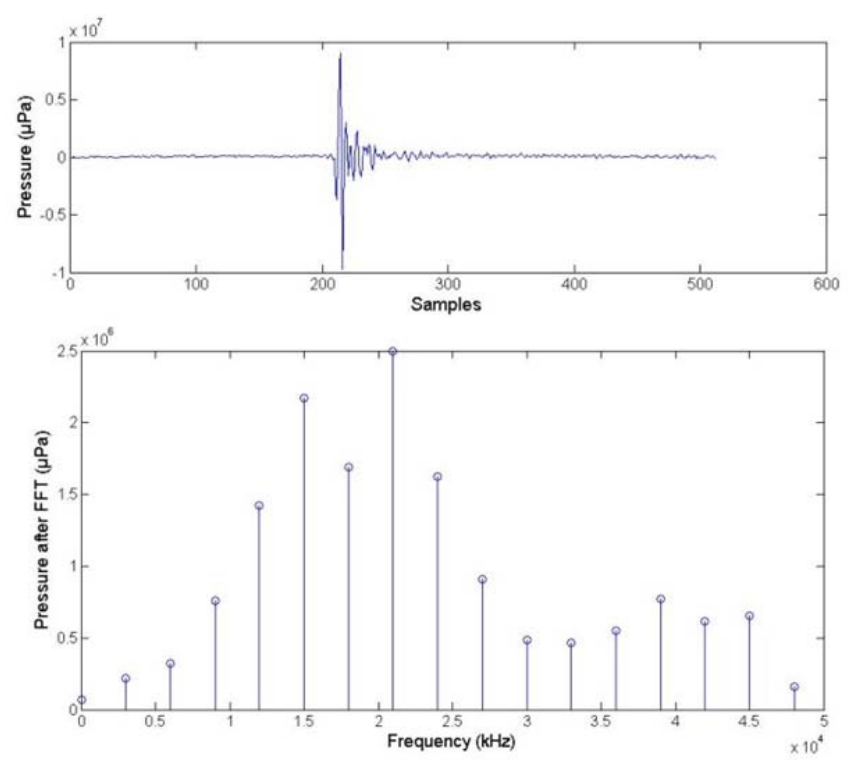

Figure 15. Temporal frame and frequency sub-frame for the decline case.
Table 1. Readings received in $\mathrm{dB}$ at 1 meter from source.

\begin{tabular}{|c|c|c|c|}
\hline $\begin{array}{l}\text { Angle over } \\
\text { semicircle }\end{array}$ & $\begin{array}{c}-45^{\circ} \text { regard to } 0 \\
\text { plane }\end{array}$ & 0 & $\begin{array}{c}+45^{\circ} \text { regard to } 0 \\
\text { plane }\end{array}$ \\
\hline-90 & 186.34 & 186.19 & 186.19 \\
\hline-45 & 186.19 & 184.06 & 183.04 \\
\hline 0 & 183.77 & 193.54 & 189.06 \\
\hline 45 & 180.69 & 183.57 & 183.46 \\
\hline 90 & 186.19 & 186.99 & 185.81 \\
\hline
\end{tabular}

Table 2. Coordinate example without correction, in degrees.

\begin{tabular}{lll}
\hline \% GPST & latitude $\mathbf{N}$ & Longitude $\mathrm{E}$ \\
\hline $2013 / 09 / 1910: 21: 21.000$ & 41.17613741 & 1.765862013 \\
$2013 / 09 / 1910: 21: 22.000$ & 41.17613436 & 1.765833378 \\
$2013 / 09 / 1910: 21: 23.000$ & 41.17614067 & 1.765866606 \\
$2013 / 09 / 1910: 21: 24.000$ & 41.17614583 & 1.765843793 \\
$2013 / 09 / 1910: 21: 25.000$ & 41.17614367 & 1.765922205 \\
\hline
\end{tabular}

Table 3. Coordinate example with correction, in degrees.

\begin{tabular}{|c|c|c|}
\hline \% GPST & latitude $\mathrm{N}$ & Longitude E \\
\hline $2013 / 09 / 19$ 10:21:21.000 & 41.17608642 & 1.765954648 \\
\hline 2013/09/19 10:21:22.000 & 41.17607901 & 1.765961776 \\
\hline 2013/09/19 10:21:23.000 & 41.17608325 & 1.765958510 \\
\hline 2013/09/19 10:21:24.000 & 41.17608555 & 1.765966284 \\
\hline 2013/09/19 10:21:25.000 & 41.17608986 & 1.765958881 \\
\hline
\end{tabular}

Table 4. Example of distance.

\begin{tabular}{lc}
\hline \% GPST & Distance $(\mathbf{k m})$ \\
\hline $2013 / 09 / 19$ 10:21:21.000 & 1.309357 \\
$2013 / 09 / 1910: 21: 22.000$ & 1.310040 \\
$2013 / 09 / 1910: 21: 23.000$ & 1.310051 \\
$2013 / 09 / 1910: 21: 24.000$ & 1.3096851 \\
$2013 / 09 / 1910: 21: 25.000$ & 1.3100011 \\
\hline
\end{tabular}

Table 5. Example of data obtained from CTD.

\begin{tabular}{clccc}
\hline Day & Time & $\begin{array}{c}\text { Temperature } \\
\left({ }^{\circ} \mathbf{C}\right)\end{array}$ & $\begin{array}{c}\text { Salinity } \\
(\%)\end{array}$ & pH \\
\hline $19 / 09 / 2013$ & $10: 20: 57$ & 23.76 & 38.04 & 8.30 \\
$19 / 09 / 2013$ & $10: 21: 17$ & 23.76 & 38.04 & 8.30 \\
$19 / 09 / 2013$ & $10: 21: 37$ & 23.76 & 38.04 & 8.30 \\
$19 / 09 / 2013$ & $11: 01: 37$ & 23.79 & 38.04 & 8.29 \\
$19 / 09 / 2013$ & $12: 01: 57$ & 24.02 & 38.05 & 8.30 \\
\hline
\end{tabular}

produced because it was initially thought that the distance between receiver and generator would be sufficient to transform the cylindrical propagation into a spherical one, but it is not sufficient.

The uncertainty value in this case is 0.67 . In this case because the seafloor is flat and so the relief does not change, the value of the spreading factor is constant.

\subsection{Evaluation of the signal receipt}

The generator and reception systems are not synchronized. Therefore the received signal timing is independent on the generation. Because the sampling frequency is 96000 Samples/second, the reception file is very big and the duration of the record is 1.5 hour, even though the test time is 15 minutes. The lack of time 
synchronization between the generator and the receptor means that the post-processed signal is a critical task.

The signal processing has been made with a Matlab ${ }^{\circledR}$ application. The sequence of this application is: first to analyze every frame that arrived from the hydrophone (512 values in int16), then to locate a maximum value and finally to create a sub-frame with the 15 index values before the maximum and 16 index value after the maximum. The subframe, with 32 values, is processed with a FFT in order to determine if the maximum value is centered in $10 \mathrm{kHz}$. Figure 14 shows the temporal frame (512 values) and the FFT of the sub-frame (32 values) for the acceptation case, and the declination case is illustrated in Figure 15. The declination case can be produced by many causes such as crustaceans, fish, seaweed and bubbles, among others.

The case accepts the maximum and minimum values of the signal, and this value divided by 2 is the amplitude of the received signal.

Once the amplitude value for the acceptation case and its time are found, another Matlab ${ }^{\circledR}$ application is used to overlap the time values.

\subsection{Sensitivity value}

The last step is to assemble all contributions and calculate the sensitivity value and its uncertainty. Table 6 shows an example of the different values of the contribution to uncertainty.

The step is calculated for every point, Table 7 shows an example with some points.

The final result is that the sensitivity is $-189.25 \pm 2.95 \mathrm{~dB}$ rel $1 \mathrm{~V} / \mu \mathrm{Pa}$ at $10 \mathrm{kHz}$.

\section{CONCLUSIONS}

The article gives the basis for considering the realization of an in situ calibration of a hydrophone with nonomnidirectional sound source. The uncertainty contribution is higher in comparison to the uncertainty obtained in the laboratory which is less than $1 \mathrm{~dB}$. For this reason, it is necessary to repeat this test to improve the different contributions to uncertainty. Improvements could include incrementing the amplitude value of the source generator which lowers the contributions factors to uncertainty of source pressure and of receiver voltage.

In the last hydrophone calibration, in February 2010, the sensitivity value was $-192 \mathrm{~dB}$ rel $1 \mathrm{~V} / \mu \mathrm{Pa}$ at $10 \mathrm{kHz}$, which is inside the confidence interval.
Table 6. Contribution to uncertainty.

\begin{tabular}{lcccc}
\hline Distance $[k m]$ & $u(S L)[d B]$ & $u(R L)[d B]$ & $u(T L)[d B]$ & $U[d B]$ \\
\hline 1.362060 & 0.01 & 0.01 & 1.31 & 2.73 \\
1.400959 & 0.01 & 0.01 & 1.35 & 2.80 \\
1.402288 & 0.01 & 0.01 & 1.35 & 2.80 \\
1.438561 & 0.01 & 0.01 & 1.38 & 2.87 \\
\hline
\end{tabular}

Table 7. Sensitivity table example.

\begin{tabular}{lcc}
\hline Distance $(\mathbf{k m})$ & Sensitivity (dB) & Uncertainty (dB) \\
\hline 1.335602 & -190.17 & 3.00 \\
1.401709 & -195.89 & 2.70 \\
1.525931 & -190.92 & 2.93 \\
1.664731 & -192.31 & 3.19 \\
\hline
\end{tabular}

\section{ACKNOWLEDGEMENT}

This work was supported by the Spanish Ministry of Economy and Competitiveness under the research project: "Sistemas Inalambricos para la Extension de Observatorios Submarinos" (CTM2010-15459).

\section{REFERENCES}

[1] "IEC60565Underwater acoustics -Hydrophones -Calibration in the frequency range $0,01 \mathrm{~Hz}$ to $1 \mathrm{MHz}$, IEC, 1997.

[2] T.Takasu, RTKLIB: Open Source Program Package for RTK-GPS, FOSS4G 2009 Tokyo, Japan, November 2, 2009.

[3] R.E. Francois and G. R. Garrison "Sound absorption based on ocean measurements: Part II: Part II: Boric acid contribution and equation for total absorption" J. Acoust. Soc. Am. Vol. 72, N.6, December 1982.

[4] Aguzzi, J.; Mànuel, A.; Condal, F.; Guillén, J.; Nogueras, M.; Del Rio, J.; Costa, C.; Menesatti, P.; Puig, P.; Sardà, F.; Toma, D.; Palanques, A. "The New Seafloor Observatory (OBSEA) for Remote and Long-Term Coastal Ecosystem Monitoring". Sensors 2011, 11, 5850-5872.

[5] "Evaluation of measurement data. - Guide to expression of uncertainty in measurement", September 2008.

[6] Garcia-Benadí, A.; Cadena-Muñoz, F.J.; Sarria, D.; Sitjar, R.; Del Rio, J. "Good practice guide for C calculation". In: 5TH MARTECH International Workshop On Marine Technology. pp. 61 - 66. 0011. ISBN 978-84-616-5764-3.

[7] T. Vicenty. "Direct and inverse solutions of geodesics on the ellipsoid with application of nested equations". Survey Review XXII, 176, April 1975. 